\title{
Adaptação e validação da Escala Filadélfia de Mindfulness para adultos brasileiros
}

\author{
Amanda da Costa daSilveira - UnivesidadeFederal doRioGrandedbSul, PotoAlege, RioGrandedoSul, Brasil \\ Thiago Goms deCastro- UniversidadeFedeal doRioGrandedbSul, PatoAlege, RioGrandedbSul, Brasil \\ WilliamBarbosa Goms - UniversidadeFedaral doRioGrandedbSul, PotoAlege, RioGrandedoSul, Brasil
}

\begin{abstract}
Resumo
A literatura psicológica tem referido especial interesse ao processo psicológico de monitoramento contínuo da experiência individual, ou mindfulness Neste estudo, buscou-se adaptar e apresentar evidências de validade convergente e divergente para uma escala de autorrelato que visa a mensurar mindfulness a Escala Filadélfia de Mindfulness (EFM). Participaram da pesquisa 703 adultos brasileiros, que responderam EFM e outras três medidas relacionadas a conceitos como autoconsciência e insight. A análise fatorial indicou a presença de duas dimensões da EFM, relativas a dois componentes de mindfulness Aceitação e Awareness Essas dimensões apresentaram índices de consistência interna de 0,859 e 0,816, respectivamente. Os resultados também indicaram convergência entre o fator Awareness e os dados de autoconsciência enquanto autofoco, e divergência entre Aceitação e facetas mal-adaptativas da autoconsciência (ansiedade social e ruminação). Em consonância com a literatura que refere haver uma faceta de mindulnesssvoltada à meditação, Aceitação apresentou também correlação positiva com Insight.

Palaurasdhave Reflexão; Consciência; Psicometria; Avaliação.
\end{abstract}

\section{Adaptation and validation of the Philadelphia Mindfulness Scale among Brazilian adults}

\begin{abstract}
Current psychological research has paid special attention to the psychological process of continuous monitoring of inner experience, i.e., mindfulness. This study aimed to adapt and to present convergent and divergent validity evidence of a mindfulness self-report measure, the Philadelphia Mindfulness Scale (PMS). Data was based on the answers of 703 Brazilian adults to the adapted version of PMS and three other measures related to self-consciousness and insight. Factorial analysis presented two principal dimensions among the items of PMS, which were related to two components of mindfulness: Acceptance and Awareness. These two dimensions presented internal consistency levels of 0,859 and 0,816, respectively. Convergences among Awareness and data related to self-consciousness taken as self-focus were observed, as well as divergences among Acceptance and maladaptive facets of self-consciousness (Social Anxiety and Rumination). In consonance to the literature on the meditating aspect of mindfulness, Acceptance also presented significant positive correlation with Insight.
\end{abstract}

Keywards Reflection; Awareness; Psychometrics; Evaluation.

\section{Adaptación y validez de la Escala Filadélfia de Mindfulness para adultos brasileños}

\begin{abstract}
Resumen
La literatura psicológica ha tenido especial interés en el proceso psicológico de monitoreo continuo de la experiencia individual, 0 mindfulness. En este estudio, se buscó adaptar y presentar evidencias de validez convergente y divergente para una escala de autorrelato que tiene por objeto mensurar mindfulness, la Escala Filadelfia de Mindfulness (EFM). Participaron de la investigación 703 adultos brasileños, que respondieron la EFM y otras tres medidas relacionadas a conceptos como autoconsciencia e insight. El análisis factorial indicó la presencia de dos dimensiones de la EFM, relativos a dos componentes de mindfulness: Aceptación y Awareness Esas dimensiones presentaron índices de consistencia interna de 0,859 y 0,816, respectivamente. Los resultados también indicaron convergencia entre el factor A wareness y los datos de autoconsciencia como autofoco, y divergencia entre Aceptación y facetas mal adaptativas de la autoconsciencia (ansiedad social y ruminación). En consonancia con la literatura que refiere haber una faceta de mindfulness vuelta a la meditación, Aceptación presentó también correlación positiva con Insight.

Palabrasdave Reflexión; Consciencia; Psicometria; Evaluación.
\end{abstract}

O consenso em torno das definições operacional e conceitual de mindfilness é alvo de extensa discussão na psicologia contemporânea (Bishop \& cols., 2004). 0 conceito foi originalmente extraído da psicologia budista e das práticas de meditação, representando, de forma abrangente, um fator mental que pode estar presente ou ausente em um dado momento de consciência (Rosch, 2007). Na tradição budista, seu significado está atrelado à clareza e flutuação nãoavaliativa da atenção mental à experiência de transição consciente momento a momento (Kabat-Zinn, 2003).
Na literatura psicológica atual, mindfiuness é considerada diferente dos estados conscientes de autoconceito, esquemas, narrativas e crenças, pois entende-se que tais conceitos passam por um crivo de juízo reflexivo. Sendo assim, a diferença de mindfiulness para processos cognitivos baseados em autorreflexão, como a autoconsciência, é a preocupação com a qualidade da experiência consciente no momento de sua ocorrência, ao contrário dos conteúdos reflexivos por ela tematizados (Shear \& Jevning, 1999).

Cientistas cognitivos têm buscado definir mindfilness de um modo que 0 torne viável 
operacionalmente para 0 treino e a mediçäo homogêneas (Van Dam, Earleywine \& Borders, 2010). Dentro de uma versão contemporânea de mindfiulness em que aspectos atencionais e de vigília da experiência presente são enfatizados, destaca-se 0 processo em seu valor de correlação com pensamentos e sentimentos adaptativos ou mal-adaptativos. Nessa linha, estudos recentes têm evidenciado a relação entre mindfulness e aspectos psicológicos tais como bem-estar subjetivo (Brown \& Ryan, 2003) ruminação (Frewen, Evans, Maraj, D ozois \& Partridge, 2008), e afeto negativo (Nyklicek \& Kuijpers, 2008). Além disso, padrões de mindfulness medidos por escalas, têm sido associados a atividade cerebral (Creswell, Way, Eisenberger, \& Lieberman, 2007), bem como resultados de terapia comportamental baseados em intervenção por meditação (Michalak, Heindenreich, Meibert \& Shulte, 2008).

No papel específico de técnicas de meditação, mindfulness foi introduzida às práticas psicoterápicas ocidentais a partir dos anos 1970 (Boorstein, 1983; Deatherage, 1975). Já nos anos 1990, observa-se um movimento de incorporação da disciplina de mindfulness às práticas psicoterapêuticas inovadoras, o que, para alguns autores (Hayes, 2004; Teasdale, Segal \& Williams, 1995), representaria uma nova onda nas chamadas psicoterapias comportamentais, sucedendo os avanços incorporados pela primeira revolução cognitiva dos anos 1950-60. Essa nova abordagem encabeçou psicoterapias baseadas no treinamento de mindfulness como por exemplo a Redução de Estresse Baseada em Mindfulness de Kabar-Zinn (2003), e a Terapia Cognitiva Baseada em Mindfulness de Teasdale, Segal e Williams (1995); e nas intervenções que incluem mindfulness enquanto um componente-chave do tratamento, como por exemplo a Terapia de Comprometimento e Aceitação de Hayes, Strosahl e Wilson (1999), e a Terapia Comportamental Dialética de Linehan (1993).

0 desenvolvimento de terapias com base na mindfulnessevidenciou um crescimento no interesse pela produção de dados empíricos relacionados ao tema dentro da academia (Cardaciotto, Herbert, Forman, Moitra \& Farrow, 2008). Contudo, o que se observa é um aumento nas produções científicas relacionadas ao tema de forma mais acelerada do que 0 amadurecimento do conceito propriamente dito (Bishop \& cols., 2004). Sendo assim, frequentemente as apresentações de medidas do construto na literatura são acompanhadas de um debate sobre a operacionalização do conceito (Brown \& Ryan, 2003; Walach, Buchheld, Buttenmüller, Kleinknecht \& Schmidt, 2006, por exemplo) ou, ainda, guardam uma certa dependência dos domínios nos quais surgiram
(Baer, Smith \& Allen, 2004; Lau \& cols., 2006, por exemplo).

Uma das definições contemporâneas de mindfiulness prioriza os aspectos atencionais e de vigúlia da experiência que estão ocorrendo no presente. O peracionalmente, esta é a definição utilizada na escala Mindful Attention Awareness Scale (MAAS - Brown \& Ryan, 2003). Os autores do instrumento relatam que 0 mecanismo autorregulatório da atenção para estados de consciência presentes é o ponto inicial e central para se compreender mindfilness e, por conseguinte, estabelecer conexões com o bem-estar psicológico. 0 instrumento, composto por 15 itens, é unifatorial. No artigo de validação, os autores indicaram que outro componente tradicional de mindfiulness relacionado às disposições de atitude - como paciência, aceitação e confiança - foi excluído da medida. Trata-se, portanto, de um instrumento fundado na autorregulação atencional para estados presentes.

Outro instrumento que se propõe a mensurar mindfulness é o Friburg Mindfulness Inventary (FMI Walach \& cols., 2006). O FMI é um inventário unidimensional em inglês construído na Alemanha. 0 instrumento tem uma forma longa, de 30 itens, específica para indivíduos familiarizados com a prática meditativa, e outra sucinta, de 14 itens, cujo uso foi indicado a não-praticantes de meditação. Os autores sugerem seu uso para avaliar intervenções clínicas e para mensurar mindfulness como traço de personalidade. Entretanto, sua faceta unidimensional não revela que facetas de mindfulness estão sendo abordadas, nem há clareza na definição operacional do conceito no artigo de divulgação da escala.

Em relação aos instrumentos produzidos com 0 intuito de investigar mindfulness em contextos aplicados específicos, destacam-se três escalas desenvolvidas na América do Norte: 0 Kentudky Inventary of Mindfulness Skills (KIMS) (Baer \& cols., 2004), o Five Fact Mindfulness Questionnaire(FFMQ) (Baer \& cols., 2006) e a Taranto Mindfulness Scale(Lau \& cols. 2006). O KIMS e o FFMQ foram construídos dentro da abordagem da Terapia Comportamental Dialética, que concebe mindfulness como um conjunto de habilidades interrelacionadas. No KIMS, são alvo de mensuração as facetas observação, descrição, ação com consciência e aceitação sem julgamento. No FFMQ, a faceta nãojulgamento da experiência interna foi acrescentada às quatro anteriores, compondo a medida que oferece cinco escores para mensurar os componentes de mindfulness

A Taranto Mindfulness Scale(Lau \& cols., 2006) foi desenvolvida para avaliar o resultado da meditação em indivíduos envolvidos em um programa de redução de estresse baseado em mindfulness $\mathrm{O} s$ autores validaram 0 
instrumento com 42 itens divididos em dois fatores (Curiosidade e Descentração), compostos a partir de itens sobre regulação atencional e qualidade da atenção não-elaborativa, caracterizada por curiosidade, aceitação e abertura à experiência. Todos os itens referem-se à descrição de uma sessão de meditação realizada imediatamente antes da aplicação da escala. Os resultados obtidos pelos pesquisadores sugerem que altos escores de descentração predizem bons resultados no programa terapêutico.

Críticas têm sido apresentadas aos instrumentos de autorrelato que visam mensurar mindfulness A abordagem de mindfulness preconizada pela MAAS, por exemplo, tem sido questionada recentemente (Cardaciotto \& cols., 2008; Rosch, 2007) por fundamentar-se excessivamente na regulação atencional e deixar de lado o componente tradicional budista da experiência de mindfulness a aceitação. Conforme Cardaciotto e cols. (2008), a aceitação seria o segundo componente de mindfulness necessário para indicar a forma como a consciência do momento presente seria conduzida. $\mathrm{Na}$ aceitação seriam contempladas as atitudes de abertura, não-julgamento e até mesmo compaixão em relação à própria experiência.

Além disso, a validade de face dos instrumentos que se propõem a mensurar mindfulness também tem sido questionada (Rosch, 2007). Brown e Ryan (2003) consideram 0 construto de mindfulness como unipolar em sua escala MAAS. Já Baer e cols. (2006) indicam uma composição de cinco facetas para o construto no FFMQ . Apesar de validar a multidimensionalidade do construto, Rosch (2007) indica que apenas um dos fatores está atrelado à observação detalhada da experiência, característica do mindfulness sendo que os quatro fatores restantes se identificam mais com uma análise de níveis de patologia. A autora sugere que essas escalas estejam medindo outros aspectos psicológicos latentes, que não mindfulness Van Dam e cols. (2010) avaliam que novas escalas de mindfulness devem ser criadas para melhor representar o construto, além de descobrir novos benefícios associados ao processo.

Neste contexto, a Philaddphia Mindfiulness Scale (PMS) foi apresentada recentemente (Cardaciotto \& cols., 2008) com o intuito de esclarecer a posição de mindfulness enquanto um processo psicológico passível de mensuração. Os autores levaram em consideração o trabalho de operacionalização do constructo de Bishop e cols. (2004), o qual sugere uma definição operacional para mindfulness baseada em dois componentes principais: uma atenção sustentada para 0 momento presente e uma atitude de abertura, curiosidade e aceitação (Bishop \& cols., 2004). Em um levantamento dos conceitos de mindfulness Cardaciotto e cols. (2008) também evidenciaram que a maior parte das definições consideravam "a consciência do momento presente" como um componente de mindfulness Consciência (Awareness), nesse sentido, foi entendida como monitoramento contínuo dos eventos internos e externos e os autores da escala preferiram o uso desse termo ao uso do termo atenção, por entenderem que este último não contemplaria todo 0 escopo experiencial, ao privilegiar, por definição, determinados aspectos da experiência em detrimento dos demais.

0 segundo componente integrante da definição operacional de mindfulness descrito na PMS refere-se à forma com que se conduz o momento presente de consciência: uma instância sem julgamentos e com atitude de aceitação em relação às experiências. Aceitação tem sido definida como a experiência dos eventos, quer externos ou internos, tais quais eles são, livres de defesas, crenças ou quaisquer outros tipos de julgamentos, elaborações ou interpretações (Cardaciotto \& cols., 2008). Para os autores, essa faceta de mindfulness coloca o conceito novamente em contato direto com sua tradição budista, que entende a renúncia e o desapego como formas de se cessar 0 sofrimento.

Desse modo, a PMS visa acessar mindfulness enquanto o contínuo monitoramento da experiência individual, sem conteúdos de julgamento. A escala apresenta, portanto, duas dimensões: "Awareness", que se refere ao monitoramento que se faz da experiência interna ou externa no momento em que elas ocorrem; e "Aceitação", que se refere a uma atitude de nãojulgamento ou de abertura relativos à experiência. Segundo os autores, altos níveis de Avarenss indicam uma capacidade alta de monitoramento individual sobre as próprias experiências internas e externas individuais, ao passo que altos níveis de Aceitação são caracterizados por uma experiência de eventos de forma completa e sem defesas, e baixos níveis são caracterizados por evitação e tentativas de controle da experiência interna (Cardaciotto \& cols., 2008).

No Brasil, observa-se 0 movimento de profissionais em disseminar a prática de mindfulness nas terapias cognitivas e comportamentais (Vandenberghe \& Assunção, 2009; Vandenberghe \& Sousa, 2006) e na investigação de suas relações com bem-estar e qualidade de vida quando atrelada à prática meditativa (Menezes \& D ellAglio, 2010). Contudo, até o presente momento inexistem medidas de autorrelato para mindfulness adaptadas para a população brasileira. Por essa razão, e considerando-se as características da PMS enquanto uma escala que oferece clareza e inovação na mensuração de mindfulness como processo psicológico, 0 presente trabalho teve como objetivo adaptar a PMS para o uso no Brasil. A versão brasileira foi chamada de 
Escala rlladeltia de IMnnulumess (EFMI). Us autores optaram por manter 0 construto mindfilness não traduzido, dada a dificuldade de correspondência do termo na língua portuguesa e em consonância com os demais trabalhos já produzidos no Brasil sobre 0 assunto.

Os objetivos específicos deste trabalho foram os seguintes: 1) Verificar a validade fatorial da EFM. A hipótese é de que a versão para o português brasileiro também apresenta dois fatores, indicando a divisão entre os componentes Awareness e Aceitação; 2) Verificar o índice de consistência interna da EFM e de suas subescalas (alfa de Cronbach); 3) Por fim, buscar indícios de validade convergente entre as subescalas da EFM e variáveis teoricamente relacionadas aos construtos. Sendo assim, espera-se encontrar correlação positiva entre a faceta Awareness de mindfulness e 0 conceito de autoconsciência privada (Fenigstein, 1997), correlações negativas entre Auareness e dimensões mal-adaptativas da autoconsciência (como os construtos de Ruminação e Ansiedade Social); espera-se também encontrar indício de correlação positiva entre Insight e o componente Aceitação da EFM.

\section{Adaptaçãoda escala}

\section{Método}

A escala foi primeiramente traduzida do inglês norte-americano para o português brasileiro por dois pesquisadores de forma independente, sendo um deles autor deste trabalho e o outro com conhecimento técnico e ciente dos objetivos da pesquisa. Nesta etapa, enfatizou-se a tradução conceitual e não estritamente literal dos itens da escala. Ambas as traduções foram comparadas e realizaram-se modificações para fins de se obter uma única tradução consensual.

Logo após a tradução consensual, um psicólogo norte-americano com fluência na língua portuguesa brasileira e que desconhecia a escala original proferiu uma tradução reversa (badk trandation) dos itens. Por fim, realizou-se uma revisão por comitê de especialistas brasileiros (fluentes em inglês) para averiguar se a versão traduzida refletia a versão original. Para todos os itens, ao menos dois juízes concordaram com a correspondência de sentido entre a tradução e 0 original, sugerindo a equivalência semântica (gramática e vocabulário) e idiomática/ conceitual (expressões com sentidos equivalentes) entre os itens. A versão preliminar do instrumento foi então aplicada em 20 participantes voluntários, que foram questionados a respeito de possíveis dificuldades encontradas para responder 0 instrumento, o que não foi constatado. Sendo assim, a versão final do instrumento foi composta e sua traduçao pode ser conferida na Tabela 1.

\section{Participantes}

Os instrumentos foram aplicados a uma amostra de conveniência de 703 adultos brasileiros predominantemente das regiões sul e sudeste do país (respectivamente 69\% e 20\% dos respondentes). 0 nordeste brasileiro teve uma representatividade de $5 \%$ da amostra, a região centro-oeste, $1 \%$ e 0 norte brasileiro contou com menos de $1 \%$ da amostra. Além disso, 4\% dos respondentes estavam residindo no exterior no momento da pesquisa. A idade média dos respondentes foi de 30,27 anos ( $\mathrm{DP}=10,63)$, sendo $62 \%$ mulheres. Do total de participantes, $38,4 \%$ eram alunos de pós-graduação, 29,3\% estavam cursando 0 ensino superior, $26,7 \%$ possuíam o ensino superior completo e 5,5\% cursaram até o ensino médio. 0 restante dos participantes $(4,8 \%)$ não informou sua escolaridade.

\section{Instrumentos}

Um breve protocolo requisitando dados demográficos foi apresentado aos participantes, em que se perguntava o sexo, a idade, o grau de escolaridade e a unidade federativa de residência do respondente. Além disso, foram aplicadas três escalas de autorrelato, além da Escala Filadélfia de Mindfiulness (EFM), cuja adaptação é o objeto desta pesquisa. Todas as medidas de autorrelato solicitam que os participantes indiquem 0 quanto se identificam com as afirmações constantes nos itens utilizando uma escala do tipo Liket de cinco pontos, em que " 0 " representa "nada característico" e "4" "extremamente característico". Os três outros instrumentos de autorrelato utilizados neste estudo são descritos a seguir:

Escala de Autoconsciência Revisada (EAC-R Scheier \& Carver, 1985): tradicional escala de autoconsciência, composta por 22 itens que visam a avaliar variações da disposição natural humana de se tornar objeto da sua própria consciência (autoconsciência). A EAC-R distingue esses pensamentos em relação a seus aspectos privados (subescala de Autoconsciência Privada (ACPR), com nove itens) e públicos (subescala de Autoconsciência Pública (ACPB), com sete itens, e subescala de Ansiedade Social (AS), com seis itens). Exemplos de itens: "Eu estou sempre tentando me entender" para $\mathrm{ACPR}$, "Eu me preocupo com a maneira como me apresento" para ACPB, e "É difícil para mim trabalhar quando há alguém me olhando" para AS. A versão adotada na pesquisa foi a traduzida e validada para a população brasileira por Teixeira e Gomes (1995). 
Questionário de Ruminação e Reflexão (QRR) (Trapnell \& Campbell, 1999): questionário que visa acessar duas formas de autoconsciência privada: reflexão (modalidade de autofoco motivada por um genuíno interesse nas informações sobre si) e ruminação (produção de modos mal-adaptativos de reflexividade). Consiste em um questionário composto por 24 itens. Metade dos itens refere-se a sentenças de reflexão, como por exemplo, "Eu adoro analisar por que eu faço as coisas", e a outra metade dos itens está relacionada à ruminação, como "Minha atenção está frequentemente focada em aspectos sobre mim sobre os quais eu gostaria de parar de pensar". Esse questionário foi traduzido e adaptado para a população brasileira por Zanon e Teixeira (2006).

1) Escala de Autorreflexão e Insight (EAI): escala australiana criada por Grant, Franklin e Langford (2002), originalmente composta por 20 itens, sendo oito itens referentes à dimensão Insighte 12 , à dimensão Autorreflexão. A EAI foi criada, segundo os autores, com a proposta de um aprimoramento da tradicional EAC aplicada aos processos de autorregulação. Insight foi definida como a clareza que o indivíduo acredita ter sobre seus próprios pensamentos, sentimentos e comportamentos, e autorreflexão foi definida como a habilidade de se interessar sobre pensamentos sobre si mesmo (Grant \& cols., 2002).

\section{Procedimentos}

Os participantes foram recrutados a partir de convites enviados por correio eletrônico à rede de contatos do grupo de pesquisa dos autores. Os destinatários eram convidados a responder a pesquisa e a sugenir o convite para seus conhecidos que se enquadrassem nos requisitos da pesquisa, seguindo, assim, a técnica de amostragem de bola de neve.

O s instrumentos, todos autoadministráveis, foram disponibilizados na internet através de um sítio atrelado à Universidade e ao sistema de coleta de dados online provido pela empresa Qualtrics <www.qualtrics.com>. O tempo médio para respondê-los foi de aproximadamente 15 minutos. Antes de responder aos instrumentos, os participantes eram solicitados a ler 0 Termo de Consentimento Livre e Esclarecido e, ao final, em caso de concordância, eram redirecionados para o preenchimento dos mesmos. O sistema do servidor Qualtrics encarregou-se de disponibilizar de forma aleatória a apresentação sequencial das quatro escalas (EFM, EAC, QRR e EAI), logo após 0 preenchimento dos dados demográficos por parte de cada um dos participantes. Ao final do preenchimento, os participantes eram redirecionados a uma tela de agradecimento e encerravam-se suas respectivas sessões de coleta de dados.

\section{Análisedos dados}

Para se verificar a composição fatorial da EFM, realizou-se inicialmente a verificação das propriedades métricas por meio dos níveis Kaiser-Meyer-Olkin (KMO), para medir a adequação da amostra, e o teste de esfericidade de Bartlett. Em seguida uma análise de componentes principais, com rotação promax, foi conduzida. 0 método de rotação oblíqua foi escolhido, pois permite que os fatores se correlacionem, levando à produção de fatores teóricos mais significativos (Netemeyer, Bearden \& Sharma, 2003). A etapa seguinte consistiu da realização da análise fatorial exploratória com o método de extração por eixos principais e rotação promax. Para avaliar a fidedignidade, utilizou-se 0 índice de consistência interna alfa de Cronbach. Correlações de Pearson foram utilizadas na análise das interações entre as subescalas da EFM e demais variáveis avaliadas, para fins de se obterem dados de validade convergente e discriminante.

\section{Resultados}

\section{Análisedecomponentes pincipais da EFM}

Nas análises preliminares, o KMO obtido para a escala foi de 0,866 e o teste de esfericidade de Bartlett $\mathrm{X}^{2}=4634,454, \mathrm{~g}=190$ e $\mathrm{p}<0,001$. Na extração de componentes principais os resultados apresentaram quatro fatores com valor superior a 1, compondo uma variância explicada de 54,871\%. Dentre os quatro fatores, apenas o primeiro e o segundo tinham autovalores superiores a 1,5. Floyd e Widaman (1995) argumentam que 0 uso de autovalores superiores a 1, como critério para manutenção de fatores extraídos, pode levar a uma hiperestimação do número de fatores a serem retidos. Em nossas análises 0 fator I apresentou autovalor de 4,628 e o fator II, valor de 3,833. Juntos, os fatores I e II explicaram $42,301 \%$ da variância total. Em observação ao scre pld, evidenciaram-se dois fatores nítidos, a partir dos quais estabeleceu-se uma linha horizontal estável para os demais fatores.

\section{Análisefatorial exploratónia}

Para examinar a validade de construto da EFM foi realizada uma análise fatorial exploratória (AFE) através do método de extração dos eixos principais, com rotação promax. Tendo em vista o observado na análise de componentes principais, optou-se na AFE pela retenção prévia de dois fatores na matriz. 0 fator I, nomeado Aceitação, agregou 10 itens e respondeu por 20,302\% da variância comum, enquanto o fator II, nomeado Awareness agregou os outros 10 itens, e explicou 15,931\% da variância. 
Os itens se distribuíram de forma homogênea entre os dois fatores, sem que houvesse necessidade de manipular os valores de carga fatorial para a composição das subescalas. Os itens carregaram exclusivamente em suas subescalas, com um valor mínimo de 0,431, conforme pode ser observado na Tabela 1.

Tabela 1. Carga componencial dos itens nos fatores I e II / rotação promax

\begin{tabular}{|c|c|c|c|}
\hline Itens & & I & II \\
\hline \multirow[t]{10}{*}{ Aceitação } & 2. Eu tento me distrair quando sinto emoções desprazerosas. & 0,431 & \\
\hline & 4. Há aspectos sobre mim mesmo sobre os quais eu não quero pensar. & 0,606 & \\
\hline & $\begin{array}{l}\text { 6. Eu tento ficar ocupado para evitar que pensamentos e sentimentos me venham à } \\
\text { mente. }\end{array}$ & 0,688 & \\
\hline & 8. Eu gostaria de poder controlar minhas emoções mais facilmente. & 0,512 & \\
\hline & 10. Eu digo pra mim mesmo que não deveria ter certos pensamentos. & 0,684 & \\
\hline & 12. Há coisas sobre as quais eu tento não pensar. & 0,783 & \\
\hline & 14. Eu digo a mim mesmo que não deveria me sentir triste. & 0,568 & \\
\hline & $\begin{array}{l}\text { 16. Se há algo em que não quero pensar, eu tento fazer várias coisas para tirar isso } \\
\text { da minha mente. }\end{array}$ & 0,753 & \\
\hline & 18. Eu tento deixar os meus problemas fora de minha mente. & 0,466 & \\
\hline & 20. Quando lembro de algo ruim, eu tento me distrair para fazer aquilo ir embora. & 0,677 & \\
\hline \multirow[t]{10}{*}{ A warenesc } & 1. Eu estou ciente de quais pensamentos estão passando em minha mente. & & 0,529 \\
\hline & $\begin{array}{l}\text { 3. Quando falo com outras pessoas, estou ciente de suas expressões corporais e } \\
\text { faciais. }\end{array}$ & & 0,476 \\
\hline & 5. Quando tomo banho, estou ciente de como a água corre sobre meu corpo. & & 0,448 \\
\hline & 7. Quando estou alarmado, percebo o que ocorre dentro de meu corpo. & & 0,558 \\
\hline & 9. Quando ando pela rua, tenho consciência dos cheiros e do ar tocando meu rosto. & & 0,533 \\
\hline & $\begin{array}{l}\text { 11. Quando alguém me pergunta como estou me sentindo, posso identificar minhas } \\
\text { emoções facilmente. }\end{array}$ & & 0,564 \\
\hline & $\begin{array}{l}\text { 13. Tenho consciência dos pensamentos que estou tendo quando meu humor } \\
\text { muda. }\end{array}$ & & 0,602 \\
\hline & $\begin{array}{l}\text { 15. Eu percebo mudanças dentro de meu corpo, como meu coração batendo mais } \\
\text { rápido ou meus músculos ficando tensos. }\end{array}$ & & 0,541 \\
\hline & $\begin{array}{l}\text { 17. Sempre que minhas emoções mudam, imediatamente eu me torno consciente } \\
\text { delas. }\end{array}$ & & 0,695 \\
\hline & $\begin{array}{l}\text { 19. Quando falo com outras pessoas, estou consciente das emoções que } \\
\text { experiencio. }\end{array}$ & & 0,656 \\
\hline
\end{tabular}

\section{Análisedefidedignidade}

0 alfa de Cronbach obtido para o fator I foi de 0,859, e para o fator II obteve-se 0 valor de alfa 0,816. Esses valores sugerem boa consistência para as subescalas. $\mathrm{Na}$ escala original, os valores de alfa obtidos para as subescalas foram iguais aos obtidos em nosso estudo: 0,85 para Aceitação e 0,81 para A wareness

\section{Evidânias devalidadeconvergnteedvergnte}

Ao se procederem análises correlacionais de Pearson entre as variáveis mensuradas pelas subescalas da EFM, EAC, QRR e EAI, pode-se compor o quadro de correlações ilustrado pela Tabela 2. Com base em Bisquerra, Sarriera e Martínez (2004), algumas correlações que merecem destaque por estarem consideradas em nossas hipóteses iniciais foram classificadas. Os escores de Autoconsciência Privada (ACPR) e Pública (ACPB), e reflexão mensurada tanto pelo QRR (REF) quanto pela EAI (ARE), correlacionaram-se de forma positiva somente com a subdimensão Awareness da EFM, tendo sido as correlações foram consideradas moderadas entre ACPR, REF, ARE e Awareness Essa correlação é considerada uma evidência de que o componente Avareness da mindfulness avalia traços que guardam semelhanças com o processo individual de autofoco em pensamentos sobre si mesmo. Por outro lado, 0 componente Aceitação apresentou correlações negativas alta e moderada, respectivamente, com duas subescalas que se propõem a medir facetas relativas a traços mal-adaptativos do autofoco: Ruminação (RUM) e Ansiedade Social (AS). Esses achados indicam a validade discriminante do fator Aceitação como um 
componente positivo e adaptativo da mindfulness Além disso, a correlação alta encontrada também deste componente com a subescala Insight reflete indícios de validade convergente da subescala de Aceitação.

Tabela 2. Correlações entre as subescalas A ceitação e Avarenesse demais escalas

\begin{tabular}{lcc}
\hline Subescala (Escala) & Aceitação (EFM) & Avarenesc (EFM) \\
\hline Autoconsciência Pública (EAC) & $-0,113^{*}$ & $0,340^{*}$ \\
Autoconsciência Privada (EAC) & $-0,167^{*}$ & $0,185^{*}$ \\
Ansiedade Social (EAC) & $-0,302^{*}$ & $-0,083$ \\
Reflexão (QRR) & $0,145^{*}$ & $0,287^{*}$ \\
Ruminação (QRR) & $-0,434^{*}$ & $-0,018$ \\
Autorreflexão (EAI) & 0,017 & $0,346^{*}$ \\
Insight(EAI) & $0,473^{*}$ & $0,218^{*}$ \\
\hline
\end{tabular}

*Correlações de Pearson com p<0,001 (2-taileed).

\section{Discussão}

O processo de adaptação cultural da EFM mostrou-se eficaz para com os objetivos do estudo. Infere-se que a ausência de maiores complicações no processo de tradução reflita também a motivação inicial dos autores da escala original em produzir um instrumento simples, que mensurasse mindfilness de forma acessível a adultos e não especificamente a indivíduos com experiência na prática meditativa (Cardaciotto \& cols., 2008).

As propriedades da EFM versão português brasileiro mostraram-se semelhantes às do instrumento original, evidenciando-se também a adequação da estrutura fatorial com a confirmação da hipótese da existência de dois componentes de mindfulness A wareness e Aceitação. Os resultados da análise fatorial exploratória apresentaram uma distribuição homogênea dos itens entre dois fatores, os quais, por sua vez, carregaram exclusivamente em um fator com valor mínimo de 0,431, indicando uma clara distinção entre os dois componentes e não sendo necessária a exclusão de nenhum item. Os dois fatores apresentaram índices de fidedignidade satisfatórios, com valores de alfa idênticos ao da escala original. A partir desses achados, aconselha-se 0 uso da escala adaptada para adultos brasileiros com 20 itens, com duas subescalas, composta por um número bem distribuído de itens, sendo 10 para o componente Aceitação e 10 para A wareness

A avaliação preliminar de validade convergente e discriminante refletiu a tendência à bidimensionalidade da EFM, indicando a convergência entre os achados referentes ao fator Auareness e os dados de autoconsciência enquanto autofoco em pensamentos e sentimentos voltados para o self, e a divergência entre 0 comportamento da subescala de Aceitação e subescalas que mensuram facetas mal-adaptativas ou tendências evitativas da autoconsciência, como é o caso da subescala de Ansiedade Social da EAC e Ruminação do QRR. Além disso, Aceitação mostrou alta correlação positiva com a subescala de Insight, evidenciando similaridades já previstas na teoria sobre mindfulness e sua faceta voltada à meditação (Kabat-Zinn, 2003).

\section{Considerações finais}

Diante das evidências de validade e confiabilidade da EFM e suas subescalas, os autores recomendam 0 uso dessa versão brasileira composta por 20 itens para pesquisas no Brasil. Os autores ainda ressaltam a predominância de indivíduos com alto índice de escolaridade (mais de $90 \%$ da amostra frequenta ou já frequentou um curso superior) que compuseram a amostra de adaptação da escala, sugerindo estudos futuros que investiguem se a EFM apresenta os mesmos índices em amostras de adultos com escolaridade em nível fundamental ou médio.

Além disso, sugere-se que se realizem estudos investigando as características psicométricas do instrumento em populações específicas, como praticantes de meditação, para fins de aprimoramento dos dados de validade da EFM. Também é recomendado que se realizem estudos futuros especificando o comportamento da EFM nas populações das regiões Norte, Nordeste e CentroO este do Brasil, dado que neste estudo prevaleceram os dados provenientes das regiões Sul e Sudeste. Ademais, a realização de estudos com outros tipos de validade e confiabilidade também são sugeridos, como validade empírica e análises de teste-reteste.

\section{Referências}

Baer, R. A., Smith, G. T., \& Allen, K. B. (2004). Assessment of mindfulness by self-report: the Kentucky Inventory of Mindfulness Skills. Assessment, 11, 191-206.

Baer, R. A., Smith, G. T., Hopkins, J., Krietemeyer, J., \& Toney, L. (2006). Using self-report assessment 
methods to explore facets of mindfulness. Assessment, 13, 27-45.

Bishop, S. R., Lau, M., Shapiro, S., Carlson, L., Anderson, N. D., Carmody, J., Segal, Z. V., Abbey, S., Velting, D., \& D evins, G. (2004). Mindfulness: a proposed operational definition. Clinical Psychdogy: Saienceand Practice, 11(3), 230-241.

Bisquerra, R., Sarriera, J. C., \& Martínez, F. (2004). Introuluãa à etatística. Enfoqueinformático como pacte estatísticoSPSS. Porto Alegre: Artmed.

Boorstein, S. (1983). The use of bibliotherapy and mindfulness meditation in a psychiatric setting. Jaumal of Transpessonal Psychdogy, 15, 173-179.

Brown, K. W., \& Ryan, R. M. (2003). The benefits of being present: mindfulness and its role in psychological well-being. Jaumal of Pesconality and Social Psychdogy, 84, 822-848.

Cardaciotto, L., Herbert, J. D., Forman, E. M., Moitra, E., \& Farrow, V. (2008). The assessment of present-moment awareness and acceptance: the Philadelphia Mindfulness Scale. Assessment, 15(2), 204-223.

Creswell, J. D., Way, B. M., Eisenberger, N. I., \& Lieberman, M. D. (2007). Neural correlates of dispositional mindfulness during affect labeling. PsychoscmaticMeeliane, 69, 560-565.

Deatherage, G. (1975). The clinical use of "mindfulness" meditation techniques in shortterm psychotherapy. Jaumal of Transpessonal Psychdogy, 7, 133-143.

Fenigstein, A. (1997). Self-consciousness and its relation to psychological mindedness. Em M. McCallum, W. E. Piper (Eds.), Psydhdogical mindeeness a contemparary undestanding(pp. 105-131). Mahwah, NJ: Lawrence Erlbaum.

Floyd, F. J., \& Widaman, K. F. (1995). Factor analysis in the development and refinement of clinical assessment instruments. Psychdogical Assesment, 3, 289-299.

Frewen, P. A., Evans, E. M., Maraj, N., D ozois, D. J. A., \& Partridge, K. (2008). Letting go: mindfulness and negative automatic thinking. Cognitive Theapy andReserrch, 32, 758-774.

Grant, A. M., Franklin, J., \& Langford, P. (2002). The Self-Reflection and Insight Scale: a new measure of private self-consciousness. Social Behavior and Pessonality, 30, 821-836.

Hayes, S. C. (2004). Acceptance \& commitment therapy, relational frame theory, and the third wave of behavioral and cognitive therapies. Behavior Therapy, 35, 639-665.

Hayes, S. C., Strosahl, K., \& Wilson, K. G. (1999). Accetance and commitment therapy. Nova Iorque: Guilford Press.

Kabat-Zinn, J. (2003). Mindfulness-based interventions in context: past, present, and future. Clinical Psychdogy: ScienceandPradice, 10, 144-156.

Lau, M. A., Bishop, S. R., Segal, Z. V., Buis, T., Anderson, N. D., \& Carlson, L. (2006). The Toronto Mindfulness Scale: development and validation. Jaumal of Clinical Psychdoy, 62, 14451467.

Linehan, M. M. (1993). Cognitivebdhavioral treatment of bordedine personality disarder. Nova Iorque: G uilford Press.

Menezes, C. B., \& DellAglio, D. D. (2010). Por que meditar? A experiência subjetiva da prática de meditação. Psicoloja emEsudb 14(3), 565-573.

Michalak, J., Heidenreich, T., Meibert, P., \& Schulte, D. (2008). Mindfulness predicts relapse/ recurrence in major depressive disorder after mindfulness-based cognitive therapy. Jamal of Nevous and Mental Disase 196(8), 630-633.

Netemeyer, R. G., Bearden, W. O., \& Sharma, S. (2003). Scaling procelures issues and applications London: Sage Publications.

Nyklicek, I., \& Kuijpers, K. F. (2008). Effects of mindfulness-based stress reduction intervention on psychological well-being and quality of life: is increased mindfulness indeed the mechanism? Annals of Behavioral Medione, 35, 331-340.

Rosch, E. (2007). More than mindfulness: when you have a tiger by the tail, let it eat you. Psydhdogical Inquiry, 18(4), 258-264.

Scheier, M. F., \& Carver, S. C. (1985). The SelfConsciousness Scale: a revised version for use with general populations. Jaumal of Appliøe Social Psychdogy, 15, 687-699.

Shear, J., \& Jevning, R. (1999). Pure consciousness: scientific exploration of meditation techniques. Em F. J. Varela \& J. Shear (Eds.), The view from within (pp. 189-209). Thorverton, Ingleterra: Imprint Academics.

Teasdale, J. D ., Segal, Z., \& Williams, J. M. G. (1995). How does cognitive therapy prevent depressive relapse and why should attention control (mindfulness) training help? Benaviar Resetch \& Therapy, 33, 25-39. 
Teixeira, M. A. P., \& Gomes, W. (1995). Selfconsciousness scale: a brazilian version. Psychdogical Repats, 77, 423-427.

Trapnell, P. D., \& Campbell, J. D. (1999). Private selfconsciousness and the five-factor model of personality: Distinguishing rumination from reflection. Jaumal of Pessonality and Social Psychdogy, 76, 284-304.

Van Dam, N. T., Earleywine, M., \& Borders, A. (2010). Measuring mindfulness? A item response theory analysis of the Mindful Attention Awareness Scale. Pesconality and Individual Differeneses 49, 805-810.

Vandenberghe, L., \& Assunção, A. B. (2009). Concepções de mindfulness em langer e KabatZinn: um encontro da ciência ocidental com a espiritualidade oriental. ContextosClínicos 2(2), 124135.
Vandenberghe, L., \& Sousa, A. C. A. (2006). Mindfulness nas terapias cognitivas e comportamentais. Reista Brasilera de Terapias Cognitivas 2(1), 35-44.

Walach, H., Buchheld, N., Buttenmüller, V., Kleinknecht, N., \& Schmidt, S. (2006). Measuring mindfulness - the Freiburg Mindfulness Inventory (FMI). Pesonality and Individual Differenes, 40, 15431555.

Zanon, C., \& Teixeira, M. A. P. (2006). Adaptação do Questionário de Ruminação e Reflexão (QRR) para estudantes universitários brasileiros. Interação emPsicoloja, 10, 75-82.

Reedidbem10/ 12/ 2010

Reformuladoem18/ 04/ 2011

Aprovadb em10/ 05/ 2012

Sobre os autores:

Amanda da Costa da Silveira é psicóloga pela Universidade Federal do Rio G rande do Sul (UFRGS) e doutora em Psicologia pelo Programa de Pós-G raduação em Psicologia da UFRGS, com estágio de doutoramento no Institute of Simulation and Training da University of Central Florida. Áreas de concentração de pesquisa: autoconsciência, cognição, linguagem e resolução de problemas.

Thiago Gomes de Castro é psicólogo pela Universidade Federal do Paraná (UFPR), mestre em Psicologia pela Universidade Federal do Rio Grande do Sul (UFRGS) e doutorando no Programa de Pós-Graduação em Psicologia da UFRG S (Bolsista CNPq/ SWE). Membro do Laboratório de Fenomenologia Experimental e Cognição (LaFEC UFRG S). Áreas de concentração em pesquisa: consciência, fenomenologia, fundamentos e medidas.

William B. Gomes é um dos fundadores do Programa de Pós-Graduação em Psicologia da UFRGS, e também 0 fundador e primeiro editor da Reista Psicdoja Rellexão eCńtica Interessa-se pela utilização de múltiplos métodos para 0 estudo de fenômenos psicológicos, com atenção à perspectiva de primeira e de terceira pessoa. É bolsista de produtividade do CNPq (1A).

Contato com os autores:

Rua Jacicoema, 492 - CEP 91770-420 - Guarujá - Porto Alegre-RS.

E-mail: amandadacosta@gmail.com 